\title{
THE OCCURRENCE OF EPILEPTIC FITS IN LEUCOTOMIZED PATIENTS RECEIVING CHLORPROMAZINE THERAPY
}

\author{
BY \\ D. W. LIDDELL and N. RETTERSTÖL \\ From Runwell Hospital, near Wickford, Essex
}

In recent years, chlorpromazine has played an increasingly important role in the treatment of mentally disturbed patients, but a number of complications and side-effects after this treatment have been recognized. The more important are hypotension, tachycardia, jaundice, leucopenia, fever, irritation at the site of injection, dermatitis, and photosensitivity. Digestive symptoms are constipation and increase in appetite and weight. Incontinence and frequency of micturition may occur, and Parkinson-like syndromes have been described.

Another complication which is not so commonly recognized is the occurrence of epileptic fits. AntonStephens (1954) mentioned a patient who had had a leucotomy and while on chlorpromazine treatment developed generalized convulsions. However, he considered them to be post-leucotomy phenomena. Lehmann and Hanrahan (1954) noted that a patient who had been given $2 \mathrm{ml}$. nikethamide after a collapse following chlorpromazine treatment developed status epilepticus. Giacobini and Lassenius (1954) observed that three of their patients, who had never had epileptic fits before, had such attacks during the treatment; one of these patients had undergone a leucotomy and another had had repeated courses of electro-shock therapy. Bonafede (1955), who claimed good results by treating chronically disturbed epileptics with chlorpromazine, mentioned one case where serial epileptic seizures, believed to have been precipitated by chlorpromazine, resulted in death. He felt that it was not usually necessary to reduce anticonvulsant therapy to give chlorpromazine, in fact, difficulty was encountered in some cases when an attempt was made to reduce the barbiturate. Merlis (1955), who had also used chlorpromazine in the treatment of severely disturbed psychotic epileptic patients, described one case of epilepsy in which convulsions were produced immediately after a chlorpromazine injection. He stated that he had been able to produce convulsions in about $2 \%$ of his 150 epileptic patients, using chlorpromazine.
Lomas, Boardman, and Markowe (1955) described epileptic fits as a complication after chlorpromazine treatment. They found epileptic fits occurred in 10 of their 800 chlorpromazine-treated mental hospital patients $(1.3 \%)$, and that this complication had no relationship either to the dosage at the time or the duration of the treatment. Six of their 10 patients had had a leucotomy, and four of these had had fits immediately after the operation, but not for many years. They concluded that chlorpromazine had epileptogenic properties which might lead to manifest fits in susceptible people.

Schlichther, Bristow, Schultz, and Henderson (1956) described the occurrence of 14 seizures in a group of 11 patients out of a total of 21 psychiatric cases which were receiving high doses of chlorpromazine. Five of these 11 cases had had a previous leucotomy and one further case had had a craniotomy.

In this paper we propose to describe seven further cases, of which six have been investigated by electroencephalography. In all, 75 chronic mental hospital patients have been treated with chlorpromazine in doses varying from 100 to $600 \mathrm{mg}$. daily. Twenty-one of these had had a prefrontal leucotomy performed at an earlier stage, and were patients who still showed behaviour disorder after the operation. In seven cases epileptic fits occurred during chlorpromazine treatment, all in the leucotomy group. The seven patients had also been treated with E.C.T. at an earlier stage, but so had also all the other patients who had not had a leucotomy. In these seven patients, one of whom died in status epilepticus, the epilepsy seemed to bear a strong relationship to chlorpromazine medication. In five cases no spontaneous fits had been experienced by the patients before the onset of chlorpromazine medication. The operation had been performed one to four years previously in these patients.

Of the seven cases with epileptic fits, six have been investigated by electroencephalography. In three of these cases it has been possible to show a 
clear-cut epileptic focus of sharp waves and spikes by phase reversal arising in the frontal lobes over the site of leucotomy. It is postulated that the cortical scar, as a result of the leucotomy operation, is potentially epileptic and is activated by chlorpromazine.

\section{Case Reports}

Case 1.-R. N. C., a man aged 41 years, has been suffering from schizophrenia since 1939. A course of cardiazol treatment was given in 1939 without effect. Bilateral prefrontal leucotomy was performed on January 3,1944. There were no complications after the operation and no epileptic fits. He had four courses of E.C.T. in 1949. Chlorpromazine treatment by mouth was given between May and July, 1954, inclusive.

A further course of chlorpromazine treatment was started on October 26, 1955, with a daily dose of $200 \mathrm{mg}$., which was increased on October 29 to $300 \mathrm{mg}$. daily. He had had $900 \mathrm{mg}$. chlorpromazine when on October 30 he experienced an epileptic fit. Neurological examination shortly after revealed no pathological findings. Chlorpromazine treatment was continued after a few days' rest; the daily dose was then increased to $400 \mathrm{mg}$. until the treatment was discontinued on February 3, 1956. No more fits occurred. Clinical improvement was obtained.

In this case the E.E.G. was recorded before the start of the first course of chlorpromazine treatment and again after the epileptic fit. It was normal on the first occasion, but after the fit the E.E.G. revealed well marked bilateral sharp wave foci by phase reversal in both frontal lobes. When the last record was taken he was still on chlorpromazine therapy.

Case 2.-H. F. N., a man aged 40 years, has been suffering from schizophrenia since 1942. Bilateral prefrontal leucotomy was performed on March 6, 1944. There were no complications after operation and no epileptic fits. A good improvement resulted and he was discharged and was able to return to work. He was re-admitted in 1948 after a relapse but recovered without treatment and was discharged after three months in hospital and again started work. He was re-admitted in 1951 and given a course of 11 electro-shocks in the same year, with some improvement. A further course of E.C.T. was given from December 1951, to January, 1952, with some resultant improvement.

Chlorpromazine treatment was started on October 17, 1955, and discontinued on February 3, 1956, after 38,000 mg. chlorpromazine had been given by mouth. Clinically a good improvement resulted. However, 11 days later he developed a typical epileptic fit. After the fit he had bilateral extensor plantar reflexes. The patient has had no further fits. He has now started a new course of chlorpromazine treatment.

An E.E.G. record taken on the same day as the fit revealed frequent paroxysms of high-voltage bilaterally synchronous $2-3 \mathrm{c}$./sec. activity, maximal frontally and near the midline. In addition, on the left side in the frontal region, in the neighbourhood of the leucotomy scar, there was a frequently-firing spike focus by phase reversal.

Case 3.-H. F., a man aged 37 years, has been suffering from schizophrenia since 1943. He was given 36 electroshocks in 1950 and 1951 without improvement. A bilateral prefrontal leucotomy was performed on May 10, 1952. There were no complications after the operation and no epileptic fits developed. Clinically there was no improvement.

Chlorpromazine treatment by mouth was started on June 27,1955 , with a daily dose of $100 \mathrm{mg}$. increased gradually to $300 \mathrm{mg}$. by July 7,1956 . After a total dose of $1,200 \mathrm{mg}$. chlorpromazine a generalized epileptic fit occurred. The treatment was discontinued and he has had no further fits.

An E.E.G. taken some time after chlorpromazine therapy had ceased was within normal limits.

Case 4.-D. C. S., a man aged 37 years, has been suffering from schizophrenia since 1937 . He was discharged in 1937 after "somnifaine" treatment and readmitted in 1939 when he received 12 cardiazol treatments and was again discharged. A further admission was necessary in December, 1943. In 1944 he had a course of E.C.T. with no clinical improvement. Later in 1944 he had 58 insulin comas. A bilateral prefrontal leucotomy was performed on December 11, 1948. Four days after operation he had an epileptic fit and six months later two further fits, one of which involved the left side of the body only. Since then he has been on phenobarbitone, gr. 1 nocte, and this dosage was maintained while he received chlorpromazine. Eight months later, in March, 1951, a further fit was observed.

Chlorpromazine treatment was started on October 21, 1955 , with a daily dose of $100 \mathrm{mg}$. which was increased to $300 \mathrm{mg}$. He had had a total of $1,900 \mathrm{mg}$. after eight days of treatment when he had a generalized fit, starting in the left side.

An E.E.G. was taken some time after the cessation of therapy and was within normal limits.

Case 5.-R. D., a man aged 29 years, was suffering from schizophrenia when admitted in 1947. He has had deep insulin therapy and one course of E.C.T., without improvement. A prefrontal leucotomy was performed in July, 1952, and there were no complications.

Chlorpromazine therapy started in January, 1956, and ended in May, 1956. The dosage was built up to 150 mg. q.d.s. In April, 1956, he had a major seizure.

An E.E.G. taken while still on therapy revealed a clear-cut right frontal spike focus by phase reversal.

Case 6.-M. E. M., a woman aged 56 years, has suffered from schizophrenia since 1929. In December, 1947, she had her first epileptic fit. She was given a course of four electro-shocks in December 1949. A bilateral prefrontal leucotomy was performed on January 24, 1951. There were no complications after the operation. However, in 1952 and 1953 she had two spontaneous epileptic fits. It should be noted that since 1947 she had been on phenobarbitone, gr. 1 twice a day, and this dosage was maintained during chlorpromazine treatment.

Chlorpromazine treatment was started on March 3, 
1956, and she was given $200 \mathrm{mg}$. daily. She had had 400 mg. in all when on March 5 she developed status epilepticus. The convulsions were, however, mainly right-sided. The fits were impossible to control and the patient died 12 hours later.

Case 7.-C. F., a woman aged 40 years, was admitted in 1938 suffering from schizophrenia. A course of cardiazol therapy was given in 1938. In 1941 a course of insulin treatment was given without benefit. A bilateral prefrontal leucotomy was performed in July, 1946. There were no complications after operation. A course of E.C.T. was given in 1951.

Chlorpromazine therapy was started in April, 1956, and a dose of $50 \mathrm{mg}$. t.d.s. was reached. After 14 days a major fit developed and therapy was stopped.

An E.E.G. taken 14 days later was found to be normal.

\section{Discussion}

The epileptic fits seemed to have no definite relationship either to the dosage or the duration of treatment. In one of the cases the fit occurred a few days after the treatment had been discontinued and after a total dose of $38,000 \mathrm{mg}$. chlorpromazine had been given. In the other six cases epileptic fits occurred during the course of treatment, often in the early days of medication, and in all cases, except in the patient who died in status epilepticus, there had been only one single fit, although treatment was continued or begun again in two cases. In two of the cases the fits were mainly unilateral. Lomas et al. (1955) suggested that chlorpromazine had epileptogenic properties which might lead to manifest fits in susceptible people.

Our E.E.G. findings seemed to support this suggestion. The E.E.G. showed that the disturbance arose frontally and a focus of epileptic activity was found in one or other, or both lobes. A positive epileptic finding of frontal dysfunction was found in three patients out of six who had had an E.E.G. taken. This positive result was obtained while they were still on medication. In the remaining three the records were taken some time after treatment had stopped and were negative. It would thus seem that there was a direct relationship between epileptic activity and chlorpromazine and that chlorpromazine activated the cortical scar. Merlis (1955) pointed out that after injection of $50 \mathrm{mg}$. chlorpromazine the typical pattern of epileptic activity was greatly accentuated and frequently made worse. Mayr and Lechner (1954) found that a latent epileptic focus can be activated in temporal lobe epilepsy by giving chlorpromazine. They considered chlorpromazine in epileptic psychoses and in status epilepticus as being absolutely contraindicated. Dogan, Vukadinović, and Longhino (1954) showed that the epileptic threshold to " metrazol "was considerably lowered by chlorpromazine. Turner, Bérard, Turner, and Franco (1956) mentioned that in cases with epileptic discharges, clear activation of such discharges occurred with chlorpromazine, while in the normal records no important modifications occurred. They proposed chlorpromazine as a method of activation in some cases of epilepsy.

It appears that chlorpromazine is definitely liable to produce epileptic fits, especially in patients who have undergone a leucotomy operation. This complication should be recognized and in cases of leucotomy we feel that anti-epileptic treatment should be combined with chlorpromazine therapy. This is important because chlorpromazine is often beneficial, and we suggest that the fits should be treated as ordinary fits and not as an indication for curtailing the treatment. The mechanism by which chlorpromazine, a tranquillizing drug, activates the cortical scar must remain in doubt. Perhaps, like sleep, it activates by lowering awareness.

\section{Summary}

Seven cases of epileptic fits during or immediately after chlorpromazine treatment are described. In all, 75 chronic mental hospital patients were treated with chlorpromazine; 21 of the patients had had a leucotomy performed. The epileptic fits all occurred in leucotomized patients. In five of the cases the fits occurred a few days after the treatment had started and in one case a few days after the treatment was discontinued. In two cases the fits were mainly unilateral. Five of the patients had not had epileptic fits after operation, while the remaining two had had no fits for a period varying from two and a half to four and a half years.

One patient died in status epilepticus.

Electroencephalographical investigations demonstrated that the disturbances arose frontally and a focus of epileptic activity was found in one or other or both frontal lobes.

It is suggested that in cases of leucotomy antiepileptic treatment should be combined with chlorpromazine treatment.

\section{REFERENCES}

Anton-Stephens, D. (1954). J. ment. Sci., 100, 543.

Bonafede, V. I. (1955). A.M.A. Arch. Neurol. Psychiat., 74, 158.

Dogan, S., Vukadinovic, D., and Longhino, A. (1954). Neuropsihijatrija (Zagreb), 2, no. 1-2, p. 88.

Giacobini, E., and Lassenius, B. (1954). Nord. Med., 52, 1693.

Lehmann, H. E., and Hanrahan, G. E. (1954). A.M.A. Arch. Neurol. Psychiat., 71, 227.

Lomas, J., Boardman, R. H., and Markowe, M. (1955). Lancet, 1, 1144.

Mayr, F., and Lechner, H. (1954).Wien. klin. Wschr., 66, 903.

Merlis, S. (1955). In Chlorpromazine and Mental Health. Proceedings of Symposium held under the auspices of Smith, Kline and French Laboratories, 1955, Philadelphia. Kimpton, London. Schlichther, W., Bristow, M. E., Schultz, S., and Henderson, A. L. (1956). Canad. med. Ass. J., 74, 364.

Turner, M., Bérard, E., Turner, N., and Franco, N. (1956). Electroenceph. clin. Neurophysiol., 8, 25. 